\title{
A new hybrid model using Step-wise Weight Assessment Ratio Analysis (SWARA) technique and Adaptive Neuro-fuzzy Inference System (ANFIS) for regional landslide hazard assessment in Iran
}

\begin{abstract}
Step-wise weight assessment ratio analysis (SWARA) method Adaptive neuro-fuzzy inference system (ANFIS) Geographical information system (GIS) Remote sensing Iran In recent years, Iran has experienced many landslides due to high tectonic activity, and a variety of geological and climatic conditions. This paper proposes a novel hybrid model based on step-wise weight assessment ratio analysis (SWARA) method and adaptive neuro-fuzzy inference system (ANFIS) to evaluate landslide susceptible areas using geographical information system (GIS). At first, based on an inventory map, landslide locations were randomly divided into two parts, $70 \%$ of which were used for generating the landslide hazard map and $30 \%$ of which were used for the validation of the model. Then, twelve landslide predisposing factors, such as lithology, slope angle, slope aspect, plan curvature, profile curvature, altitude, distance to streams, distance to faults, distance to roads, land use, seismicity, and rainfall were considered for the analysis. All the factors were then weighted by the SWARA method. Considering the nature of predisposing factors, they were split into two groups, factors with discrete data and factors with continuous data. For factors with discrete data, the SWARA method was used for final weight of each class, and for factors with continuous data, results related to the center of each class were obtained from the SWARA method. Subsequently, AFNIS was used to obtain weight of each value. All the values obtained from the model were then used to generate the landslide hazard map of the study area. Finally, the landslide hazard map was validated by receiver operating characteristics (ROC) using both success rate curve and prediction rate curve. $70 \%$ of observed landslides were used for the former while the remaining was used for the latter. The validation results showed that the area under the success rate curve and prediction rate curve (AUC) are 0.84 and 0.80 respectively. Additionally, the prediction performance of the SWARA method for landslide hazard mapping was investigated and the results were compared with those obtained from the proposed model. The comparison revealed that the developed model has better prediction ability for landslide hazard assessment. The results also indicated that the proposed model used in this study produced satisfactory and reliable landslide hazard map, which can be used for preliminary land use and infrastructure planning in Iran.
\end{abstract}

Keyword: Landslide susceptibility; Step-wise weight assessment ratio analysis (SWARA) method; Adaptive neuro-fuzzy inference system (ANFIS); Geographical information system (GIS); Remote sensing; Iran 